\title{
Electronic skin replaces volunteer experiments
}

\author{
N. Nessler, W. Reischer \& M. Salchner \\ Institute for Applied Physics, University of Innsbruck, Innsbruck, Austria
}

\begin{abstract}
Neutral electrodes (grounding plates used in electrosurgical operations) were tested with thermo cameras in volunteer experiments. These experiments are difficult to perform and expensive. A new test device swaroTEST with a measuring board "electronic skin" simulates the relevant electrical features of human skin to replace volunteer experiments. This device consists of a three dimensional resistor network representing the electric features of the skin and muscle tissue, and a temperature sensing array (one transistor for each $\mathrm{cm}^{2}$ ) to measure the resultant temperature increase after a standardized current load (700 $\mathrm{mA} \mathrm{hf}$ current during $60 \mathrm{~s}$, proposed in the relevant AAMI HF-18 standard). The comparison of test results with thermo camera images of volunteer experiments showed a sufficient coincidence, which proves the applicability of this test device to replace volunteer experiments.
\end{abstract}

Keywords: electronic skin, electrosurgery, neutral electrode.

\section{Introduction}

Neutral electrodes (dispersive electrodes, grounding plates) are safety relevant accessories in electrosurgery and must be tested according to AAMI HF 18 standard. A neutral electrode in contradiction to the active electrode (electrical knife) must not heat the patient's skin in excess of $6^{\circ} \mathrm{C}$ during the hf-current pass.

One test method is a volunteer experiment under standardized conditions (AAMI) with a thermo camera to measure the temperature increase on the skin under the neutral electrode. This method is laborious (10 volunteers for one significant test!), time consuming and expensive (thermo camera, clinical environment) and cannot be used routinely for quality tests. The result of a typical volunteer experiment is shown in fig. 1. As usual the higher temperatures are displayed in yellow to red, cooler sites are green to blue. 
An alternative is a suitable structured surrogate medium (AAMI) which simulates the electrical features of the patient's skin found in electrosurgery. The solution is an electronic simulation of the skin $\rightarrow$ the electronic skin.

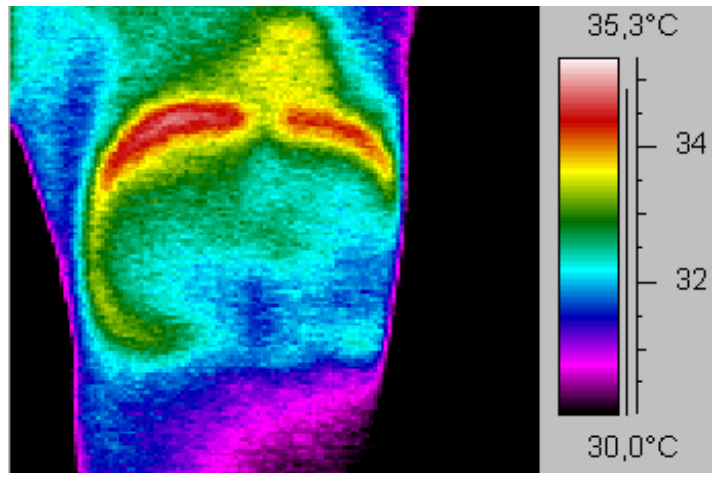

Figure 1: Thermo camera image of a volunteer experiment showing the typical edge effect.

\section{Theoretical calculation}

Human skin at the preferred locations for application of a neutral electrode (e.g. thigh, upper arm, buttock) consists of several layers with various electrical conductivities.

From the only rarely available information about electrical properties of skin in the literature $[2,3]$ the thigh-model is composed of the various skin layers, the muscle and the bone.

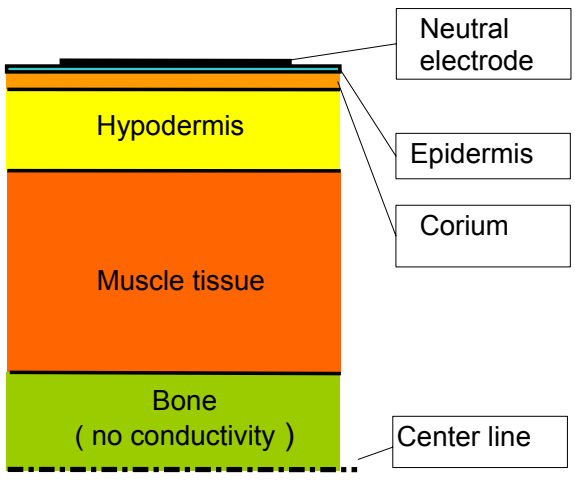

Figure 2: Skin layers of a thigh used in model calculations and as basis for the resistor network. 
In detail the model contains the epidermis $(0,1 \mathrm{~mm}$ thickness, $2000 \mathrm{Ohm} \mathrm{cm}$ specific conductivity), corium $(1,5 \mathrm{~mm}, 290 \mathrm{Ohm} \mathrm{cm})$, hypodermis with fat $(15$ $\mathrm{mm}, 1600 \mathrm{Ohm} \mathrm{cm})$, muscle $(65 \mathrm{~mm}, 200 \mathrm{Ohm} \mathrm{cm})$ and bone (no conductivity) as shown in Fig. 2. The neutral electrode for the model is a standard adult type with approximately $130 \mathrm{~cm}^{2}$ active area $(80 \times 170 \mathrm{~mm})$.

This volume conductor causes a current concentration around the edges of the neutral electrode ("edge effect") and therefor an excessive heating at the highest current densities.

The electronic simulation of the skin must therefor reproduce the current flow inside the skin and muscle tissue to generate the same energy distribution (and heating effects) as in the patient's skin.

\section{Electronic realization}

The device is realized as a stack of pcb-boards with a gold plated surface for the attachment of the neutral electrode under test. This surface is divided into $1 \mathrm{~cm}^{2}$ sized squares, each of them connected to a network of resistors at the rear side. This network corresponds to the specific impedance in horizontal and vertical direction of the respective skin layers for a prism with $1 \mathrm{~cm}^{2}$ cross section each. Fig. 3 gives an impression of this network.

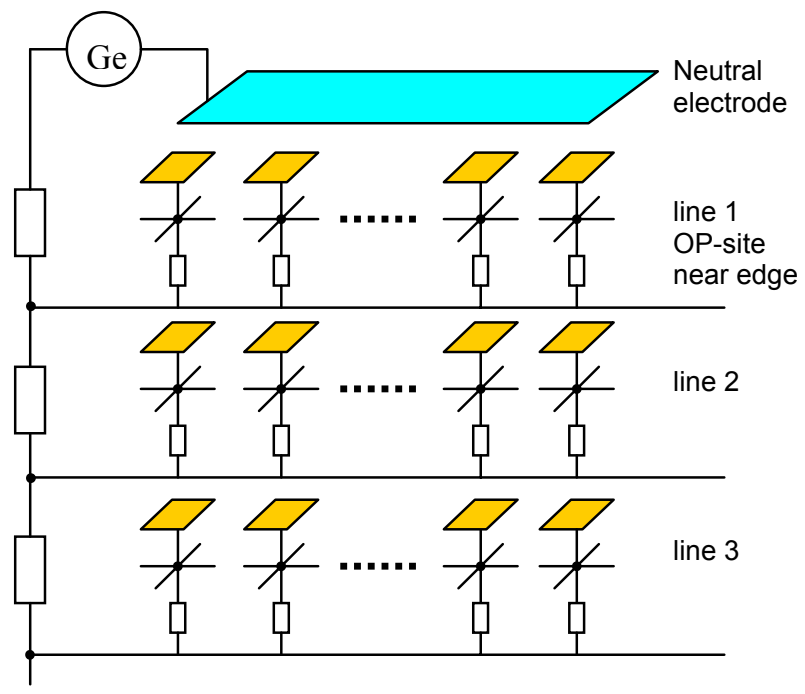

Figure 3: Principle of impedance network for skin simulation

One of the network "prisms" as symbolized in Fig. 3 is shown in more details in Fig. 4. A group of 6 resistors each represent one skin layer.

In a next development step this impedance network is simplified and optimized to correspond with the current distribution found in model calculations. The values of the impedances are dimensioned to simulate the worst case of a "thermal sensitive volunteer" (AAMI). 


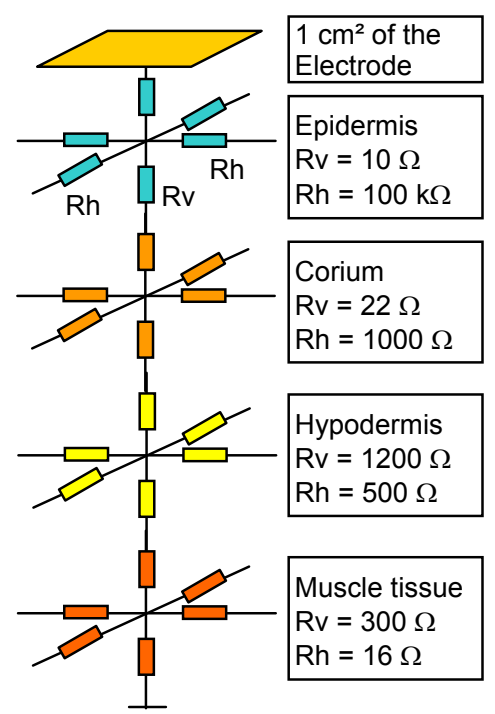

Figure 4: Three dimensional R-network for representation of the electrical features of the human skin.

For each square centimeter of resistor network a transistor as temperature sensor is in thermal contact to measure the local temperature. The temperature dependent base-emitter voltage is scanned by a computer controlled electronic. By comparing the temperature before and after the application of the standard load according to AAMI HF-18 (700 mA during $60 \mathrm{~s}$ for adult sized neutral electrodes) the relevant "temperature increase" is calculated as the difference of the local temperature values.

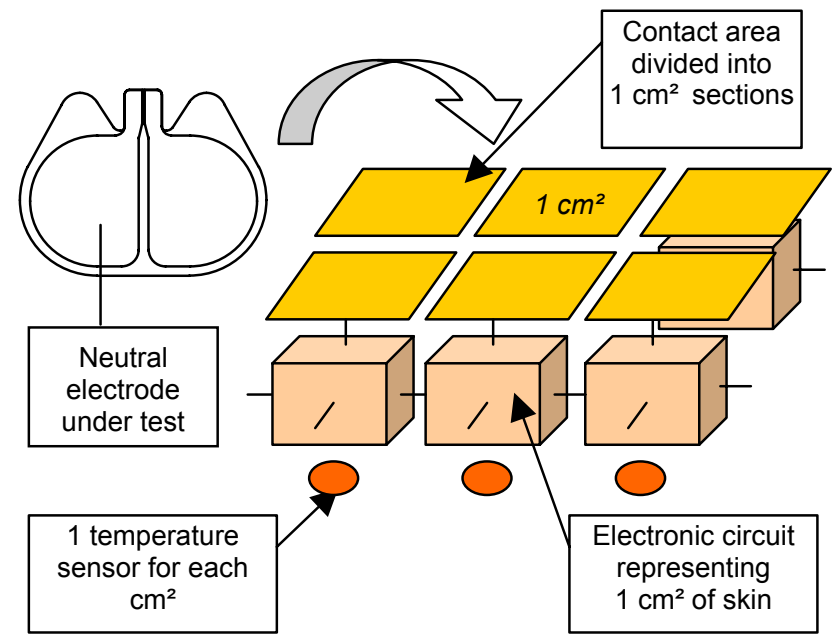

Figure 5: Measuring table and its components. 
The final device swaroTEST consists of the main unit including the frequency generator, amplifier, control logic and the serial interface for the connection to the PC and the measuring table to hold the neutral electrode under test. The measuring table has a gold plated surface with a dimension of $12 \times 22 \mathrm{~cm}$, divided into electrically separated areas of $1 \mathrm{~cm}^{2}$ each to be connected to the electronic network described above.

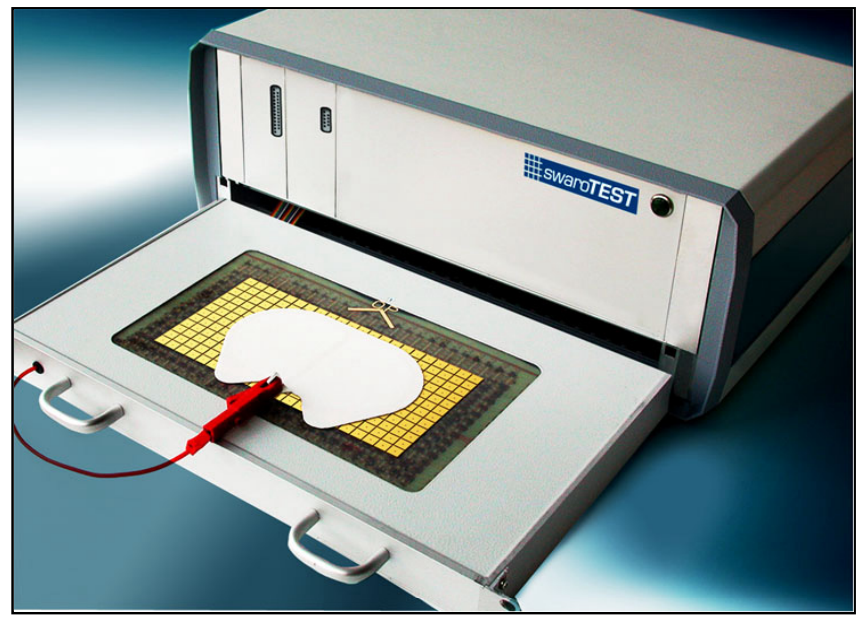

Figure 6: The device swaroTEST.

The control software is self explanatory and starts with a selection of the measuring parameters to adopt the temperature measurement to different skin properties and electrode sizes (e.g. adult electrodes need $700 \mathrm{~mA}$ for test, infant $500 \mathrm{~mA}$ ).

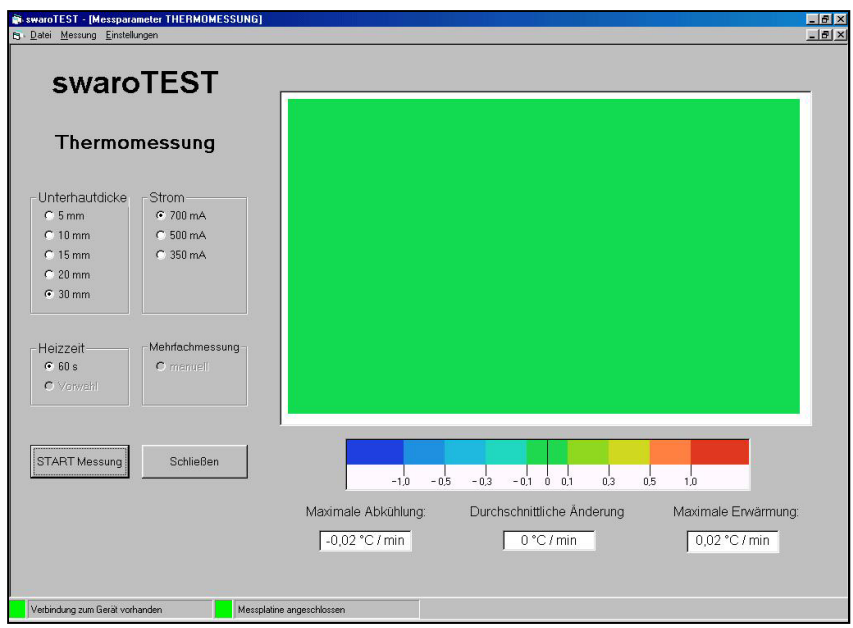

Figure 7: Starting screen with parameter selection and temperature control. 
The green field indicates the temperature increase / decrease of the measuring area, thus indicating whether after a previous measurement the surface temperature has already stabilized (thermal equilibrium) and the next measurement may be started. This uniform green field indicates "no temperature change", the next measurement can start.

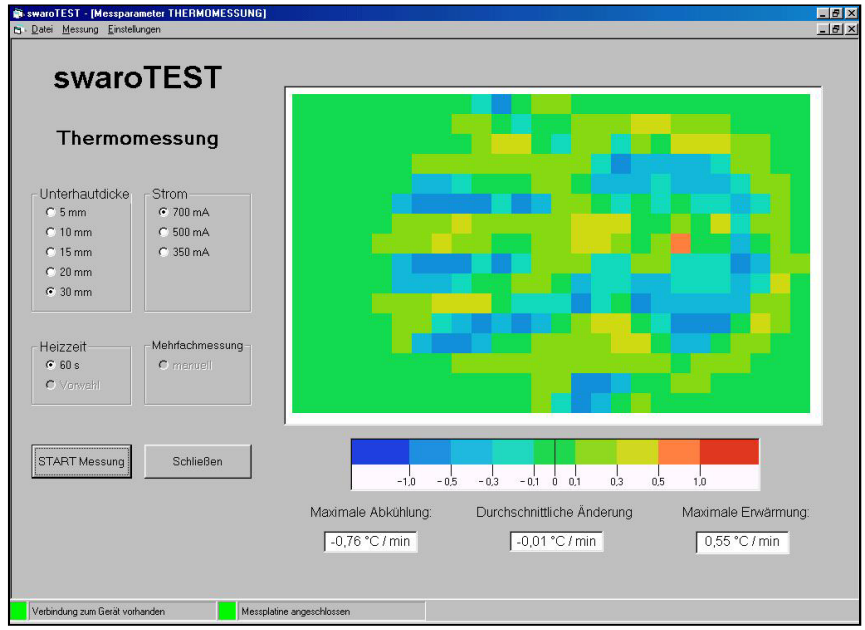

Figure 8: Starting screen indicating a cooling in progress.

Fig. 8 shows the starting screen while cooling (blue colors) and partly warming (yellow and red colors) is in progress. A measurement under this conditions would lead to false results.

The measurements itself runs fully automatically and displays the result on the PC screen. After start of measurement the current trough the neutral electrode is kept constant by a closed loop control for $60 \mathrm{~s}$. After the heating period the temperatures in the measuring transistors are scanned and the temperature difference to the "cold" measurement is calculated. A typical result is shown in fig 9 .

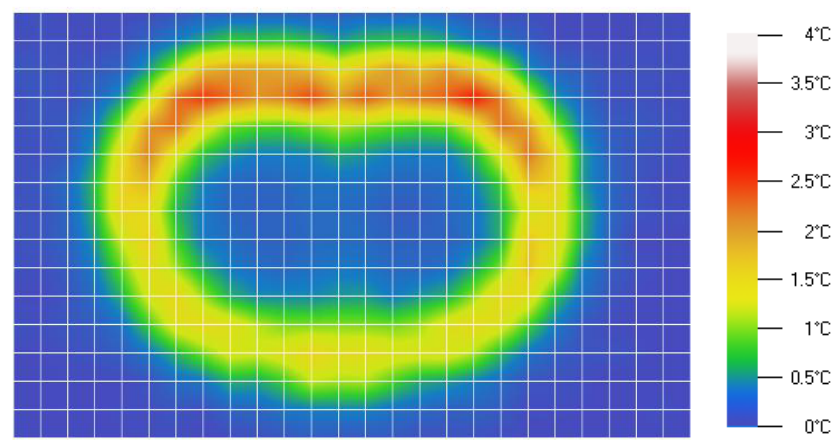

Figure 9: Result of a temperature measurement. 
Test measurements with several types of neutral electrodes under various test conditions showed a close correlation with the thermo camera images measured in volunteer experiments.

The test parameters for the result displayed in fig. 9 were similar to those found in the volunteer test in fig 1 . The coincidence of the temperature profile proves that the swaroTEST device can replace volunteer experiments.

For a more detailed view a relief representation can be selected and rotated to give an impressive view of the temperature increase.

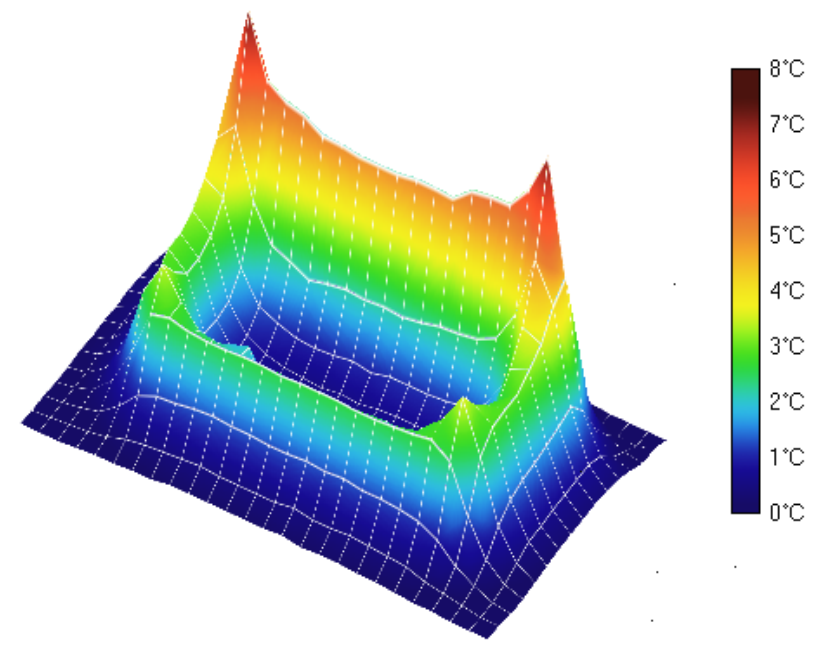

Figure 10.

\section{Results}

According to the requirements of the AAMI HF18 standard a device replacing volunteer experiments must prove to yield the same results as the volunteer experiments for comparable conditions. Therefor we have performed more than 30 volunteer experiments and compared them with the results of our swaroTEST device.

\section{Conclusions}

This new device swaroTEST is an electronic replacement for thermal tests of neutral electrodes under AAMI HF 18 conditions. The electronic skin simulates the various skin layers by electronic circuits in a way to yield the same current distribution and the same heating effect as found in volunteers. The results are reproducible and by far less laborious compared with volunteer experiments The device swaroTEST can be used for quality test in a production process and for evaluation of new designs of neutral electrodes and replace volunteer experiments. This yields reproducable results at low cost within a short time 


\section{References}

[1] American National Standard Institute ANSI/AAMI HF18-93, Association for the Advancement of Medical Instrumentation, Electrosurgical Devices, Arlington, USA, 1993

[2] Gabriel S., Lau R.W., Gabriel C., The dielectric properties of biological tissue: II Measurements in the frequency range $10 \mathrm{~Hz}$ to $20 \mathrm{GHz}$, Phy. Med. Biol. 41, 2251-2293, 1996

[3] Edrich J., Cookson C. C., Electrosurgical dispersive electrodes heat cutaneous and subcutaneous skin layers, Medical Instrumentation Vol 21, p 81-86, 1987

[4] Nessler N., Die Neutralelektrode bei der Elektrochirurgie, ein Risiko für den Patienten?, Österreichische Krankenhauszeitung ÖKZ, 12/95, p 2934, 1995

[5] Nessler N., Current density distribution in Human skin under the Grounding electrode of Electrosurgery, BEMS 17th Annual Meeting, Boston, MA., 1995

[6] Nessler N., Huter H., Wang L., Sicherheitstester für HF-ChirurgieNeutralelektroden, Biomedizinische Technik Vol 38, p 5-9, 1993

[7] Nessler N, Reischer W., The neutral electrode in electrosurgery, a risk for the patient? , MEDICON 2001, Pula

[8] Nessler N, Reischer W., Salchner M. Electronic skin - test device for electrosurgical neutral electrodes, $12^{\text {th }}$ IMEKO TC4 International Symposium, Zagreb 2002 